\title{
TTR
}

Traduction, terminologie, re?daction

\section{Jean Delisle. La Traduction raisonnée : manuel d'initiation à la traduction professionnelle de l'anglais vers le français. Les Presses de l'Université d'Ottawa, 1993, 484 p.}

\section{José Tourville}

Volume 6, numéro 2, 2e semestre 1993

Traduction, mixité, politique

URI : https://id.erudit.org/iderudit/037161ar

DOI : https://doi.org/10.7202/037161ar

Aller au sommaire du numéro

Éditeur(s)

Association canadienne de traductologie

ISSN

0835-8443 (imprimé)

1708-2188 (numérique)

Découvrir la revue

Citer ce compte rendu

Tourville, J. (1993). Compte rendu de [Jean Delisle. La Traduction raisonnée : manuel d'initiation à la traduction professionnelle de l'anglais vers le français. Les Presses de l'Université d'Ottawa, 1993, 484 p.] TTR, 6(2), 190-196.

https://doi.org/10.7202/037161ar d'utilisation que vous pouvez consulter en ligne.

https://apropos.erudit.org/fr/usagers/politique-dutilisation/ 


\section{Works cited}

Nord, Christiane (1993). Einführung in das funktionale Übersetzen. Tübingen/Basel, Francke.

Pöchhacker, Franz (1993). "From Knowledge to Text: Coherence in Simultaneous Interpreting." Yves Gambier \& Jorma Tommola, eds. Translation and Knowledge (SSOTT IV). Turku, University of Turku Centre for Translation and Interpreting, pp. 87-100.

Vermeer, Hans. J. (1992). Skizzen zu einer Geschichte der Translation. Vols. I \& II. Frankfurt/Main, Verlag für Interkulturelle Kommunikation.

Jean Delisle. La Traduction raisonnée: manuel d'initiation à la traduction professionnelle de $l^{\prime}$ anglais vers le français. Les Presses de l'Université d'Ottawa, 1993, 484 p.

Dans son article intitulé "les Manuels de traduction: essai de classification ", Jean Delisle constate que "les auteurs de manuels de traduction semblent réticents à faire figurer le mot "manuel" ou "textbook" dans le titre de leurs ouvrages» (p. 21). Et bien, cette fois, Jean Delisle, pionnier de la pédagogie de la traduction et partisan de l'enseignement par objectifs d'apprentissage, nous offre bel et bien un manuel: le titre est clair et informatif. Les buts à atteindre le sont tout autant: "Le futur traducteur apprendra donc à repérer et à nommer les principaux pièges du transfert interlinguistique,

1. TTR, $V(1), 1^{\text {er }}$ semestre 1992, pp. $17-47$ 
développera son aptitude à prévoir les solutions possibles pour les éviter et cnrichira ses moyens d'expression en langue écriten (p. 15).

L'ouvrage comporte huit chapitres qui correspondent aux objectifs généraux suivants:

$\begin{array}{ll}\text { I. } & \text { Métalangage de l'initiation à la traduction } \\ \text { II. } & \text { Documentation de base du traducteur } \\ \text { III. } & \text { Méthode de travail } \\ \text { IV. } & \text { Processus cognitif de la traduction } \\ \text { V. } & \text { Conventions de l'écriture } \\ \text { VI. } & \text { Difficultés d'ordre lexical } \\ \text { VII. } & \text { Difficultés d'ordre syntaxique } \\ \text { VIII. Difficultés d'ordre rédactionnel }\end{array}$

Chaque chapitre comprend autant de sections que d'objectifs spécifiques et ces derniers sont suivis de suggestions de lecture et d'exercices d'application' ${ }^{2}$.

En fait, la Traduction raisonnée est un mégamanuel. Même si l'auteur n'avait pas l'ambition de couvrir tous les problèmes de traduction imaginables (p. 16), l'ouvrage qu'il nous propose impressionne par son contenu diversifié. Les 56 objectifs spécifiques portent sur des sujets et des problèmes qui tous méritent d'être abordés. Il serait cependant vain de les aborder tous en un semestre ${ }^{3}$. Il le serait tout autant de les évaluer tous en quelques pages. Je me limiterai donc à quelques commentaires.

Les derniers objectifs généraux (IV à VIII) de la Traduction raisonnée renferment la plupart des objectifs de la seconde partie de

2. Le Livre du maître renferme le corrigé des 80 exercices et la traduction des 60 textes anglais.

3. Comme le confiait l'auteur lors d'un séminaire tenu à Concordia en août dernier, le manuel est conçu pour les trois premiers cours de traduction générale d'un programme professionnel. 
$l^{\prime}$ Analyse du discours ${ }^{4}$. Ceux-ci sont cependant revus et augmentés. Un seul exemple suffira, je l'espère, à souligner les améliorations. L'objectif VI de l'Analyse du discours, "l'Exégèse lexicale», traite de la traduction des termes à contenu vague et à très haute fréquence «corporate» et "pattern" ainsi que des "déterminatifs" «-minded", «-conscious" et "-oriented». L'objectif VI de la Traduction raisonnée, qui porte aussi sur les difficultés d'ordre lexical, compte 16 objectifs spécifiques. La traduction de "pattern" constitue bien l'un d'entre eux, mais il est entièrement remanié. L'exposé est rédigé en fonction du destinataire: l'étudiant. De nouveaux "correspondants» du mot "pattern" viennent s'ajouter aux anciens «équivalents lexicaux" et des exemples de traduction complètent maintenant l'exposé. Toutes les unités textuelles à traduire ont été remplacées et l'étudiant n'a plus à les traduire entièrement: il n'a qu'à substituer une équivalence idiomatique aux pointillés (tâche suffisante, vu la difficulté de l'exercice).

Tout comme dans l'Analyse du discours, l'auteur souligne l'apport de diverses approches, celui de la stylistique comparée surtout, même s'il privilégie l'approche interprétative ${ }^{5}$. Il expose différents points de vue et cite de nombreux auteurs, mais il va plus loin, cette fois: il nous fait nettement sentir que la recherche empirique en traduction est non seulement essentielle, mais bien vivante $^{6}$. En cela, la traduction raisonnée marque le début d'une ère

4. Jean Delisle, l'Analyse du discours comme méthode de traduction (Les Presses de l'Université d'Ottawa, coll. "Cahiers de traductologie", $n^{\circ} 2,1980,282$ p.). L'ouvrage, qui s'adresse autant aux apprentistraducteurs et aux pédagogues qu'aux théoriciens et aux praticiens de la traduction, comporte deux parties: «Aspects théoriques» (réflexion sur la théorie et l'enseignement de la traduction professionnelle) et «Aspects pratiques».

5. Le lecteur ne s'étonne donc pas que «les Structures résultatives» (p. 298), objectif d'ordre syntaxique qui traite du chassé-croisé, précède "Tournures nominales, tournures verbales» (p. 337), seul objectif portant sur la transposition et qui est d'ordre rédactionnel, celui-là.

6. Par exemple (p. 338): «Toutes ces affirmations - et il serait facile d'allonger la liste - ne semblent pas reposer sur des relevés statistiques. Or, une étude récente, menée par Ginette Demers, 
nouvelle. Les discussions classiques ${ }^{7}$ prennent en effet une allure tout à fait moderne. Certaines sections pourraient même à elles seules constituer un "Catalogue des idées reçues sur la traduction" ${ }^{8}$.

Les trois premiers objectifs généraux (signalés par des chiffres romains) sont très différents des derniers. L'objectif II comprend deux objectifs spécifiques (signalés par des chiffres arabes). Dans le premier, l'auteur décrit la bibliothèque de base du traducteur de textes pragmatiques de l'anglais vers le français, recense les principaux types de répertoires et autre sources documentaires, fournit des critères d'évaluation qualitative des ouvrages de référence. Les cinq exercices d'application sont bien conçus et peuvent en inspirer de nombreux autres. Le second objectif spécifique, tout aussi utile, porte sur les limites des dictionnaires bilingues mais peut également donner l'occasion d'aborder les limites des dictionnaires unilingues (ou d'autres ouvrages).

L'objectif III comporte un excellent exposé sur les étapes de la méthode de travail, sur les tâches à accomplir avant, pendant et après l'opération de traduction. S'ajoutent deux objectifs spécifiques "l'Explication de texte» et «le Repérage des difficultés de traduction" qui détaillent deux des étapes préalables au transfert interlinguistique. Le repérage des difficultés de traduction a pour but d'aider l'étudiant à reconnaître les difficultés récurrentes de traduction des textes pragmatiques. C'est un objectif central du

professeur à l'Université Laval [...].» Ou encore, (p. 421): «Les résultats d'une recherche encore inédite sur la fausse question par Suzanne Pons-Ridler et Geneviève Quillard tendent à confirmer statistiquement les observations d'Hilaire Belloc et des auteurs de la SCFA.»

7. Est-il juste de croire que les substantifs prédominent en français (pp. 337-339)? Est-il vrai que le français fait un moins grand usage du passif (pp. 330-332)? La fausse question est-elle plus fréquente en français qu'en anglais (pp. 420-421), etc.?

8. Cf. Marina Yaguello, Catalogue des idées reçues sur la langue (Paris, Éditions du Seuil, coll. «Point Virgule», 1988). Dans cet ouvrage de vulgarisation, l'auteure s'attaque à un certain nombre de préjugés sur la langue. 
manuel puisque la méthode d'initiation proposée vise justement à ce que l'étudiant puisse repérer et nommer $a$ priori les principaux pièges du transfert interlinguistique. Si l'auteur considère que l'étudiant devrait déjà avoir une idée de la traduction qu'il produira lors de la lecture du texte de départ", il n'en reste pas moins que certaines difficultés ne pourraient être repérées à la seule lecture du texte original (l'auteur fournit d'ailleurs la traduction). Les deux exercices d'application paraissent porter davantage sur l'acquisition $\mathrm{du}$ métalangage que sur le repérage des difficultés de traduction, mais l'auteur avait bien précisé: "On ne saurait être en mesure de repérer les difficultés de traduction les plus courantes des textes sans avoir au préalable assimilé la plupart des notions et catégories définies dans le glossaire placé en tête de l'ouvrage» (p. 97).

On s'y attendait, puisque Jean Delisle écrivait (1992, p. 40, cf. note 1): «la plupart des auteurs ne prennent pas toujours la peine de présenter systématiquement le métalangage essentiel à l'apprentissage raisonné de la traduction [...]. Or l'expérience prouve qu'il est difficile de tenir un discours réfléchi sur les phénomènes de la traduction ou encore d'évaluer pertinemment des traductions sans disposer d'une terminologie adéquate. Privé de ce cadre notionnel indispensable, on bascule rapidement dans l'impressionnisme stérile contraire à un enseignement universitaire de qualité. Il m'apparaît donc nécessaire de faire acquérir le métalangage de base de la pédagogie de la traduction, qu'il faut se garder de confondre avec les notions de la linguistique générale.»

Voilà qui est bien raisonné, en effet, mais on s'interroge sur l'opportunité d'exiger d'un étudiant qui ne s'est pas encore "frotté" à la pratique de la traduction qu'il assimile dès le premier objectif les cent quatre-vingt-sept notions du glossaire qui se trouve en tête de

9. "Cette opération préliminaire [la lecture] consiste aussi à repérer les difficultés de traduction et à prévoir déjà les procédés à mettre en cuvre au moment du transfert» (p. 85). 
l'ouvrage (pp. 19-49) ${ }^{10}$. Peut-on vraiment s'attendre à ce qu'il réponde dès les premières semaines de cours à des questions telles que «Peut-on dire qu'il existe un rapport entre les notions de "tonalité" et de "compensation"? Précisez.» ou encore "Qu'entend-on par le terme "cooccurrence"?» (p. 61) $)^{11}$ ?

Les questions les plus simples de ce premier objectif sont parfois les plus déroutantes. À la dernière question précitée, l'étudiant qui aura appris par cœur la définition du terme répondra que par "cooccurrence" on entend ("on" renvoyant à Jean Delisle bien sûr): "Présence dans un même énoncé de deux ou plusieurs unités lexicales distinctes, consécutives ou non et formant une unité de signification" (p. 25). Mais peut-être confondra-t-il "cooccurrence» et "collocation", et dans ce cas-là, il répondra: "Association syntagmatique dans un même énoncé de deux ou plusieurs unités lexicales distinctes et habituellement liées" (p. 23). L'enseignant souhaitera peut-être que l'étudiant ne uraisonne» pas trop cette fois ou du moins qu'il ne demande pas quelle est la différence entre "cooccurrence» et «collocation». Les exemples de l'auteur ne sont par ailleurs pas très éclairants. "Un bruit court, une rumeur circule», exemples de collocation, illustrent-ils une association plus "syntagmatique» $\mathrm{d}$ 'unités lexicales plus «liées" que «faire, débiter, dire, lire un discours", exemples de cooccurrence?

Mes critiques paraîtront sans doute sévères, mais c'est bien un manuel que Jean Delisle nous propose ici. Et même s'il précise ( $p$. 53) qu'il présente le métalangage de l'initiation à la traduction générale, le glossaire étonne. Il paraît curieux $d^{\prime} y$ voir inclus «objectif

10. L'auteur avait préalablement souligné (p. 53): «Il importe de bien assimiler ces quelque cent quatre-vingts notions fondamentales pour être en mesure de tenir un discours réfléchi sur l'opération de traduction et pour se familiariser le plus rapidement possible avec les réalités du transfert interlinguistique.»

11. On peut également s'interroger sur la pertinence de questions telles que «En quoi un "objectif général d'apprentissage" se distingue-t-il d'un "objectif spécifique d'apprentissage"?» (p. 61) ou encore «Quel est le synonyme de la figure de rhétorique appelée "personnification"?» (p. 62). 
d'apprentissage», curieux d'y voir se côtoyer "faute» et "zeugme», "adjectif qualificatif» et "déictique». Sachant que le glossaire se compose de notions empruntées à une dizaine de domaines (grammaire, linguistique générale, linguistique différentielle, analyse littéraire, etc.), le lecteur averti comprendra sans doute que certains termes communs à plusieurs disciplines n'aient pas la même aire sémantique pour les uns et pour les autres ${ }^{12}$. Néanmoins, le lecteur qui connaît bien l'Analyse du discours s'étonnera de constater que la terminologie n'est plus celle qu'elle était ${ }^{13}$. Ceci dit, le glossaire reste très utile (d'autant plus utile que l'ouvrage n'a pas d'index). L'enseignant pourra toujours passer outre à ce premier objectif ou l'aborder différemment.

La Traduction raisonnée n'en possède pas moins tous les éléments d'un excellent ouvrage. Jean Delisle offre cette fois encore matière à réflexion sur la traduction aux étudiants comme aux pédagogues, aux théoriciens comme aux praticiens.

José Tourville Université Concordia 